\title{
Catching a Moving Target: Comparative Modeling of Flaviviral NS2B-NS3 Reveals Small Molecule Zika Protease Inhibitors
}

Szymon Pach, ${ }^{1}$ Tim M. Sarter, ${ }^{2}$ Rafe Yousef, ${ }^{1}$ David Schaller, ${ }^{1}$ Silke Bergemann, ${ }^{1}$ Christoph Arkona, ${ }^{1}$ Jörg Rademann, ${ }^{1}$ Christoph Nitsche, ${ }^{2}$ Gerhard Wolber *1

${ }^{1}$ Pharmaceutical and Medicinal Chemistry, Institute of Pharmacy, Freie Universität Berlin, Königin-Luise-Straße 2+4, Berlin 14195, Germany

2 Research School of Chemistry, Australian National University, Canberra, ACT 2601, Australia

*E-mail: gerhard.wolber@fu-berlin.de

\section{Contents}

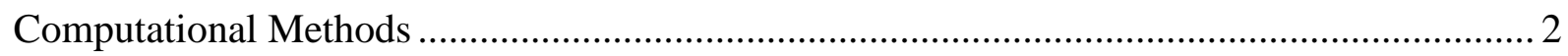

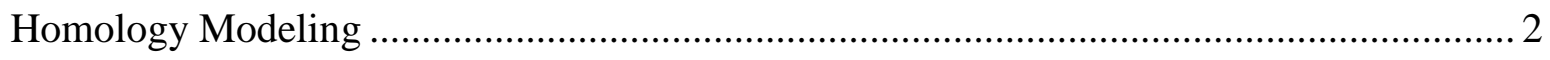

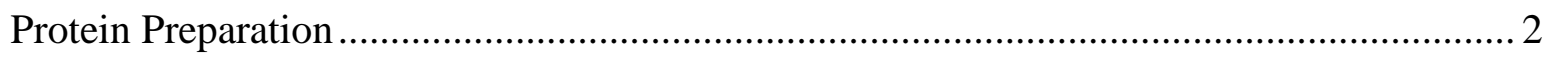

Active and Decoy Molecules for Model Development and Validation ................................ 2

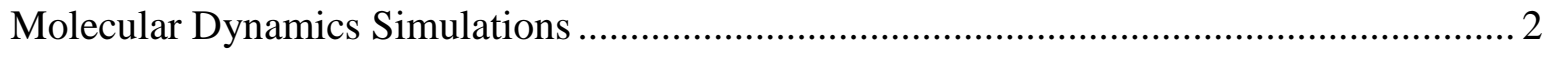

Pharmacophore Generation with PyRod .......................................................................... 3

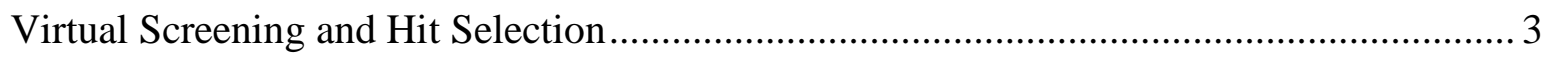

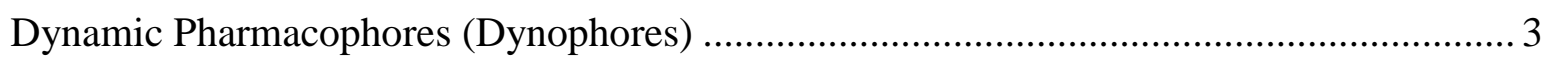

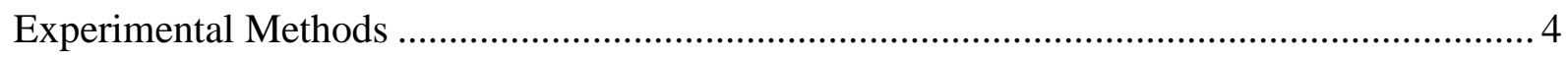

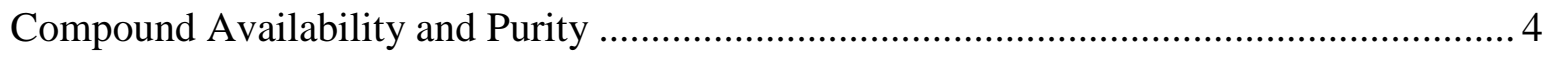

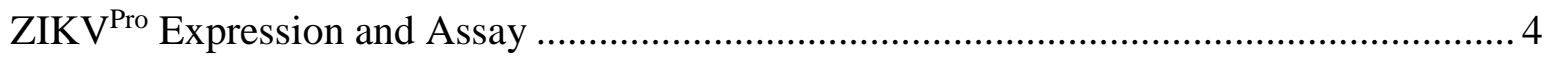

$\mathrm{WNV}^{\text {Pro }}$ and DENV2 ${ }^{\text {Pro }}$ Expression and Assay ........................................................... 4

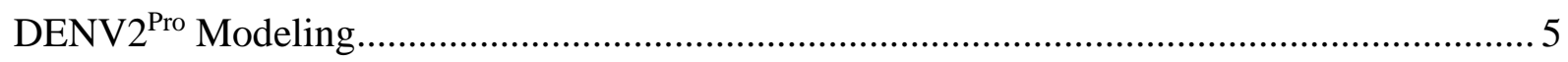

Comparison of DENV2 ${ }^{\text {Pro }}$ and $\mathrm{WNV}^{\text {Pro }}$ Binding Pockets ................................................ 5

Comparison of Inhibitor-Binding Modes with $\mathrm{ZIKV}^{\text {Pro }}$ and $\mathrm{WNV}^{\mathrm{Pro}}$................................. 5

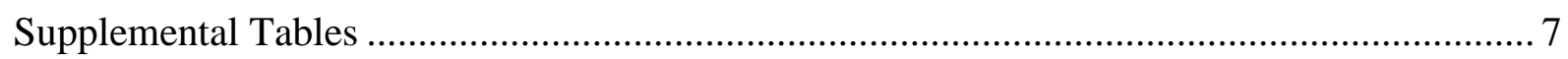

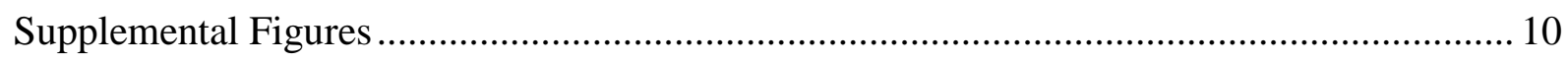

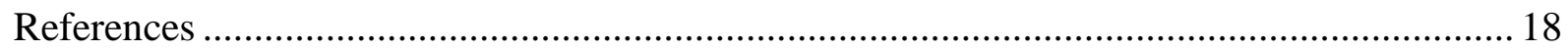




\section{Computational Methods}

\section{Homology Modeling}

Due to the lack of a high quality crystal structure of Dengue virus serotype 2 protease $\left(\mathrm{DENV} 2^{\mathrm{Pro}}\right.$ ) in the closed conformation, a homology model was prepared using MOE $2018^{1}$. The model was constructed using GB/VI scoring ${ }^{2}$ with a maximum of ten main chain models based on the closed conformation of DENV3 ${ }^{\text {Pro }} \mathrm{X}$-ray crystal structure with the best resolution (PDB ID: 3U1J ${ }^{3}$ ).

\section{Protein Preparation}

Protein structure data was obtained from the Protein Data Bank ${ }^{4}$ (PDB). We chose closed conformation X-ray crystal structures of NS2B-NS3 Zika Virus (ZIKV ${ }^{\text {Pro }}$, PDB-ID: 5YOF ${ }^{5}$ ) and West Nile Virus protease (WNV ${ }^{\text {Pro }}$, PDB-ID: $5 \mathrm{IDK}^{6}$ ) with best resolution and PDB validation scores. The crystal structures were prepared using MOE $2018^{1}$ by deleting not resolved residues, co-crystalized ligands and water molecules, capping termini and protonating the system at $\mathrm{pH}$ of 7 and temperature of $300 \mathrm{~K}$.

\section{Active and Decoy Molecules for Model Development and Validation}

A manually curated library of ligands active against flaviviral NS2B-NS3 was generated by selecting compounds from literature with $\mathrm{IC}_{50}$ or $\mathrm{K}_{\mathrm{D}}$ values lower than $50 \mu \mathrm{M}{ }^{7}$ Based on the observation that D129 plays a crucial role for ligand recognition, ${ }^{6}$ we included only ligands with cationic structures. The library contains 17 small-molecular active ligands with reported inhibition of $\mathrm{WNV}^{\text {Pro }}$ (supporting information, Table S1). No ligands matching our selection criteria were targeting $\mathrm{ZIKV}^{\text {Pro }}$. All peptides and derivatives were excluded from the validation of the combinatorial 3D pharmacophore library due to lacking drug-likeness. 667 decoy molecules (assumed as inactive on the target) were generated with the DUD-E web service $^{8}$ based on the structures of the 17 active compounds. The ligands were protonated using MOE 2018 and starting conformations were generated using Corina 3. ${ }^{9}$ Conformations for active and decoy set were generated with iCon (iCon best settings: maximal 200 conformations, root mean square threshold of 0.8 and energy window of 20.0) implemented in LigandScout $4.2 .^{10,11}$

\section{Molecular Dynamics Simulations}

MD simulations of DENV2 ${ }^{\text {Pro }}$, WNV ${ }^{\text {Pro }}$, and $\mathrm{ZIKV}^{\text {Pro }}$ were prepared using Maestro $11.7^{12}$ and performed with ten replicates for each system with Desmond $5.5^{13}$ on Nvidia GeForce GTX 1080Ti graphics cards. The prepared proteins were solvated in a cubic box with $15 \AA$ padding using TIP4P water mode ${ }^{14}$ and neutralized with sodium ions. The osmolarity of the system was adjusted using $0.15 \mathrm{M}$ sodium chloride. The system was equilibrated on default settings. The dynamics of the system were observed over 10 ns resulting in 2000 time-steps. The trajectories were wrapped and aligned on heavy atoms of the protein backbone with VMD 1.9.3. ${ }^{15}$ 


\section{Pharmacophore Generation with PyRod}

PyRod ${ }^{16}$ describes pharmacophoric binding pocket characteristics with dynamic molecular interaction fields (dMIFs) holding information about the protein environment of water molecules throughout MD simulations which are further processed to yield pharmacophore features for virtual screening. Pharmacophore models were generated using PyRod 0.7.1 by analyzing the last 5 ns (or 1000 time-steps) of the ten MD replicates for each system. The first 5 ns were retained for conformational equilibration of the protein. A rectangular grid of size 22 x 17 x $30 \AA$ centering around the catalytic serine 135 covered the binding pocket of both proteins sufficiently and was used in PyRod settings for trajectory analysis. The grid-point distance was set to $0.5 \AA$. The characteristics of exclusion volume features mimicking the protein surface were set to default. The dMIFs were transformed into an unrefined pharmacophore model with up to 20 interaction points per feature type.

Subsequently, features of the unrefined pharmacophore model placed outside the conserved binding sub-pockets S1 and S2 were removed and remaining features were prioritized according to dMIFs for each feature type. Based on this focused pharmacophore, a combinatorial pharmacophore library was created. The number of independent features combined to a pharmacophore was set to minimal 3 and maximal 6 (including up to 4 hydrogen bonds, 3 hydrophobic contacts, 3 aromatic and 3 ionic interactions). The cationic interaction with D129 was selected to be present in each generated pharmacophore. The combinatorial pharmacophore library for $\mathrm{WNV}^{\text {Pro }}$ was validated using the above-mentioned ligand sets. The pharmacophore models were prioritized for early enrichment factor $\left(\mathrm{EF}_{1 \%}\right)$ and absolute number of recovered active inhibitors. The three best performing pharmacophores were used for virtual screening of commercially available compound libraries.

\section{Virtual Screening and Hit Selection}

The virtual screening (VS) of commercially available compound libraries (Asinex, Chembridge, Chemdiv, Enamine, Key Organics, LifeChemicals, Maybridge, SPECS, VitasM each in version from year 2018) containing in total 7.6 million ligands was performed using LigandScout 4.2. To further filter pharmacophore screening hits and to obtain plausible binding hypotheses, virtual screening hits were docked into the $\mathrm{WNV}^{\text {Pro }}$ and $\mathrm{ZIKV}^{\text {Pro }} \mathrm{x}$-ray crystal structures (respective PDB-IDs are listed above) using GOLD 5.6.3 ${ }^{17}$ with 10 docking poses per ligand generating diverse solutions (ligand root mean square deviation more than $1.5 \AA$ ) with search efficiency set to $100 \%$ and $\mathrm{ASP}^{18}$ as scoring function and Chemscore ${ }^{19}$ for rescoring. The binding cavity was set as a sphere with $10 \AA$ around the side-chain oxygen atom of catalytic S135. The generated binding hypotheses were energy minimized with MMFF94 force field ${ }^{20-24}$ and filtered employing the pharmacophores used for screening and scored by the pharmacophore fit in LigandScout 4.2. The remaining compounds were simulated in a 20 ns MD on settings described above. The hits showing stable positions in the binding pocket over the course of the simulation were selected for the biochemical testing.

\section{Dynamic Pharmacophores (Dynophores)}

We used our in-house developed dynamic pharmacophore application (dynophores) ${ }^{25,} 26$ for the analysis of binding modes of the active inhibitor 427_1. The Dynophore framework automatically generates interaction density clouds via creation of 3D pharmacophores with LigandScout $^{10,11}$ for each time-step of a MD simulation. The statistical characterization of 
such interaction patterns is automatically implemented in Dynophore application including feature occurrence-frequency and distance plots. A series of MD simulations was performed to compare binding hypotheses of ligand - protease complexes. Each ligand - protease system was simulated on setting described above in five replicates over 50 ns generating in total 25,000 conformations.

\section{Experimental Methods}

\section{Compound Availability and Purity}

The selected hit compounds were purchased from Chembridge, USA (397_2: 72768922, 397_6: 38579749, 427_1: 79134560, 427_2: 59734232) and Enamine, Ukraine (397_12: Z2440305346). The purity (> 94\%) of all assayed compounds was confirmed in-house with HPLC.

\section{ZIKV Pro Expression and Assay}

The unlinked construct (bZiPro) is available at Addgene, USA (plasmid \#86846) and was expressed and purified as described previously. ${ }^{27}$ All assay measurements were performed in triplicate in $10 \mathrm{mM}$ Tris-HCl, $\mathrm{pH} 8.5,20 \%$ (v/v) glycerol, and $1 \mathrm{mM} \mathrm{CHAPS}$ as described previously. ${ }^{28}$ Various concentrations of test compounds (10 mM DMSO stocks) were pipetted into a 96-well plate (black U-bottom, Greiner Bio-One, Austria). Zika virus NS2B-NS3 protease (bZiPro) was added to a final concentration of $1 \mathrm{nM}$ and the mixture was incubated for $10 \mathrm{~min}$. Subsequently, the enzymatic reaction was initiated by adding the substrate BzNle-Lys-Lys-Arg-AMC (Biosyntan, Germany) to three final concentrations (ranging from 2.5 to $20 \mu \mathrm{M}$ ). The gradual release of fluorescent 7 -amino-4-methylcoumarin (AMC) was monitored for $70 \mathrm{~s}$ at $460 \mathrm{~nm}$ with excitation at $360 \mathrm{~nm}$, using a fluorophotometer (Spectramax M2e plate reader, Molecular Devices, USA). Initial velocities were derived from the linear curves as variation of relative fluorescence intensity per unit time. IC $\mathrm{C}_{50}$-values were calculated from at least seven different inhibitor concentrations using Prism 8.2. ${ }^{29}$ Assuming a competitive inhibition model (Cheng-Prusoff equation) ${ }^{30} \mathrm{IC}_{50}$ values were plotted against the substrate concentrations, followed by linear regression to determine $\mathrm{K}_{\mathrm{i}}$ values with coefficients of determinations $\left(\mathrm{R}^{2}\right)$ of 0.999 and 0.980 for compounds 397_2 and 427_1, respectively.

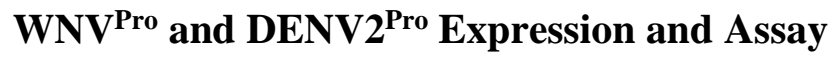

The $\mathrm{WNV}^{\text {Pro }}$ was expressed in the E. coli strain BL21 (DE3) / pWNV NS2B-NS3 transfected with a pQE-60-based expression-plasmid carrying the cDNA of the fusion protein NS2B-NS3. In this fusion protein two functional subunits, amino acids 52-96 of cofactor NS2B and amino acids 1-184 of NS3 serine protease were connected via a $\mathrm{G}_{4} \mathrm{SG}_{4}$ linker peptide. At the carboxy terminus the fusion protein carried a six-fold histidine tag. Cells were incubated at $37^{\circ} \mathrm{C}$ and shaken with $120 \mathrm{rpm}$ in the presence of ampicillin $(100 \mu \mathrm{g} / \mathrm{ml})$ until the optical density at $600 \mathrm{~nm}\left(\mathrm{OD}_{600}\right)$ had reached 0.4 . Subsequently, the cell suspension was cooled to $20^{\circ} \mathrm{C}$ and further incubated until the optical density reached 0.6. Protein expression was started by adding isopropylthiogalactoside (IPTG, $1 \mathrm{mM}$ ), and incubation was continued at $20^{\circ} \mathrm{C}$ for $16 \mathrm{~h}$. The cell culture was centrifuged at $1500 \mathrm{~g}$, and the pellet was lysed in $25 \mathrm{mM}$ Tris-HCl pH 7.5, $50 \mathrm{mM} \mathrm{NaCl}, 1 \%(\mathrm{v} / \mathrm{v})$ Triton X-100, 10\% (v/v) glycerol in an 
ultrasonic bath under cooling with ice. The suspension was clarified by centrifugation, and the NS2B-NS3 fusion protein was purified from the supernatant by NiNTA-agarose chromatography followed by gel filtration on a Superdex 75 HiLoad 16/60 column in $25 \mathrm{mM}$ HEPES-NaOH pH 8.0, $50 \mathrm{mM} \mathrm{NaCl,} \mathrm{5 \%} \mathrm{(v/v)} \mathrm{glycerol.} \mathrm{The} \mathrm{Expression} \mathrm{of} \mathrm{DENV2}{ }^{\text {Pro }}$ started from an analogous construct in the vector pET-15b (Novagen) resulting in a NS2B fusion protein with amino terminal six-fold histidine tag.

All assay measurements were performed in triplicate in either $10 \mathrm{mM}$ MOPS buffer, $\mathrm{pH} 8$, $20 \%(\mathrm{v} / \mathrm{v})$ glycerol $\left(\mathrm{WNV}^{\text {Pro }}{ }^{31}\right.$ ) or in $50 \mathrm{mM}$ Tris-HCl $\mathrm{pH} 8.5 / 20 \%$ (v/v) glycerol (for DENV2 $^{\text {Pro }}{ }^{32}$ ). Various concentrations of test compounds (15 mM DMSO stocks) were pipetted into a 384-well plate (Corning 3766, black, with clear flat bottom and non-binding surface). WNV protease was added to a final concentration of $20 \mathrm{nM}$ or $800 \mathrm{nM}$ for DENV2. Subsequently, the enzymatic reaction was initiated by adding the substrate Boc-Gly-Lys-Arg$\mathrm{AMC}^{33}$ (Bachem, Switzerland) to three final substrate concentrations (ranging from 50 to $833 \mu \mathrm{M}$ for WNV or 18.75 to $75 \mu \mathrm{M}$ for DENV2). The gradual increase of fluorescence due to the release of 7-amino-4-methylcoumarin (AMC) was monitored for $600 \mathrm{~s}$ at $465 \mathrm{~nm}$ with excitation at $360 \mathrm{~nm}$, using an Infinite M1000 plate-reader (Tecan, Switzerland). Initial velocities were derived from the linear curves and expressed as variation of relative fluorescence intensity per unit time. $\mathrm{IC}_{50}$ values were calculated from at least seven different inhibitor concentrations using Prism 6.8. ${ }^{29}$ Assuming a competitive inhibition model (ChengPrusoff equation) ${ }^{30} \mathrm{IC}_{50}$-values were plotted against the substrate concentrations, followed by linear regression to determine $K_{i}$ values with coefficients of determinations $\left(R^{2}\right)$ of 0.951 and 0.961 for compounds 397_2 and 427_1 on WNV ${ }^{\text {Pro }}$, respectively and 0.931 for compound 427_1 on DENV2 ${ }^{\text {Pro. }}$.

The pH-value of 8.0 used for the $\mathrm{WNV}^{\text {Pro }}$ assay is slightly different from pH-optimum for this protease. However, the difference should not influence the protease activity dramatically. Nall et al. ${ }^{34}$ has shown that the reaction rate for the hydrolysis of a substrate at $\mathrm{pH} 8.0$ reaches $75 \%$ of the reaction rate value at $\mathrm{pH} 8.5$.

\section{DENV2 ${ }^{\text {Pro }}$ Modeling}

\section{Comparison of DENV2 ${ }^{\text {Pro }}$ and $\mathrm{WNV}^{\text {Pro }}$ Binding Pockets}

All available DENV2 ${ }^{\text {Pro }}$ structures show the open conformation, which is hypothesized to be catalytically inactive. ${ }^{35}$ Therefore, we built a homology model of the closed conformation of DENV2 NS2B-NS3 based on related crystalized DENV3 ${ }^{\text {Pro }} .^{3}$ The binding pockets of $\mathrm{WNV}^{\text {Pro }}$ and DENV2 ${ }^{\text {Pro }}$ show high homology (Figure S3). The homology model retained stable backbone root mean square deviation (RMSD) value upon ten MD simulations used in the next step for the PyRod analysis. The focused DENV2 ${ }^{\text {Pro }}$ pharmacophore (B3, Figure S4) comprises of similar interaction patterns in homologous binding sub-pockets as in the substrate binding pocket of WNV NS2B-NS3.

Subsequently, we generated binding hypotheses for active inhibitors 397_2 and 427_1 via molecular docking (Figure S5). Both ligands showed interaction patterns predicted by PyRod.

\section{Comparison of Inhibitor-Binding Modes with ZIKVPro and WNVPro}

The active inhibitor 427_1 shows on DENV2 ${ }^{\text {Pro }}$ nearly an order of magnitude higher activity than on $\mathrm{ZIKV}^{\text {Pro }}$. To investigate this gain in activity, we performed MD simulations 
of the protease-inhibitor complex and created dynamic pharmacophores (Dynophores). The dynamic interaction patterns of 427_1 in complex with DENV2 ${ }^{\text {Pro }}$ are comparable with other proteases (A, Figure S6). Noticeable is an aromatic pi stacking between phenyl moiety of 427_1 and Y161 appearing more frequently $(37.4 \% \pm 7.7 \%)$ in comparison to ZIKV ${ }^{\text {Pro }}$ $(3.1 \% \pm 1.8 \%)$ and $\mathrm{WNV}^{\mathrm{Pro}}(2.0 \% \pm 1.5 \%)$. We assume that this interaction contributes to the stronger inhibitor binding. Additionally, we observed upon visual inspection of Dynophores that interaction density clouds in the S2 sub-pocket were less scattered than in other both 427_1-protease complexes. We inspected the trajectories of MD simulations and observed the opening of a cleft between NS3 and NS2B of DENV2 ${ }^{\text {Pro }}$ allowing good alignment of the inhibitor in the S2 sub-pocket. In order to quantify the cationic key interaction, we measured the distance between positively ionizable pyrrolidine moiety of 427_1 and carboxylate of D75 for all inhibitor-protease complexes using the Dynophore application. The distance of salt bridge in DENV2 ${ }^{\text {Pro }}$ is lower with narrower distribution compared to the other two flaviviral species (B-D, Figure S6). To rationalize the cleft opening, we used the methodology and findings of Christiane Schüler's master thesis carried out in the group of Prof. Wolber (personal communication). In this in-silico study, Christiane Schüler showed that polymorphic mutations of flaviviral proteases have a strong influence on inhibitor binding and activity. She identified the F85M mutation of NS2B as the driving force for the opening of the NS3-NS2B-cleft allowing for additional interactions between inhibitor and protease. As a descriptor for this effect, distance between the C $\alpha$-atoms of residue 85 (or homologue 84) in NS2B and G153 in NS3 was applied successfully. We measured this distance for DENV2 ${ }^{\text {Pro, }}$ ZIKV ${ }^{\text {Pro }}$, and $\mathrm{WNV}^{\text {Pro }}$ in complex with 427_1 (Figure S7). The distance distribution indicates that the probability of NS2B-NS3-cleft opening is higher in DENV2 ${ }^{\text {Pro }}$ than for the two other species, which supports our hypothesis of optimized conformational fit of the protease to 427_1.

In the next step, we investigated, why $\mathbf{3 9 7} \_2$ is less potent in DENV2 ${ }^{\text {Pro }}$ (inhibition cut-off $>50 \mu \mathrm{M})$ compared to the other proteases $\left(\mathrm{K}_{\mathrm{i} \text { WNV }}=7.4 \mu \mathrm{M}, \mathrm{K}_{\mathrm{i} \text { ZIKV}}=11.5 \mu \mathrm{M}\right)$. We surmise that the S1 sub-pocket of DENV2 ${ }^{\text {Pro }}$ is more lipophilic than in $\mathrm{ZIKV}^{\text {Pro }}$ and $\mathrm{WNV}^{\text {Pro }}$ as indicated by PyRod-derived pharmacophores (Figure S4). Inhibitor 397_2 exposes a methoxymoiety in this protein region, which is more polar compared to an analogue methyl-moiety in inhibitor 427_1, contributing to activity-loss. Additionally, the ether-moiety of inhibitor 397_2 establishes hydrogen bond to the OH group of Y161. This is only a weak hydrogen bond $^{36}$, compared to the hydrogen bond established via a carbonyl group of inhibitor 427_1 (Figure S5), contributing to the destabilization of 397_2-binding in the S1 sub-pocket. 


\section{Supplemental Tables}

Table S1. The library of active WNV ${ }^{\text {Pro }}$ inhibitors for the validation of 3D pharmacophores. *- two different $\mathrm{K}_{\mathrm{D}}$-values ( 40 and $83 \mu \mathrm{M}$ ) are reported for this compound; for more details see ref. ${ }^{37}$.






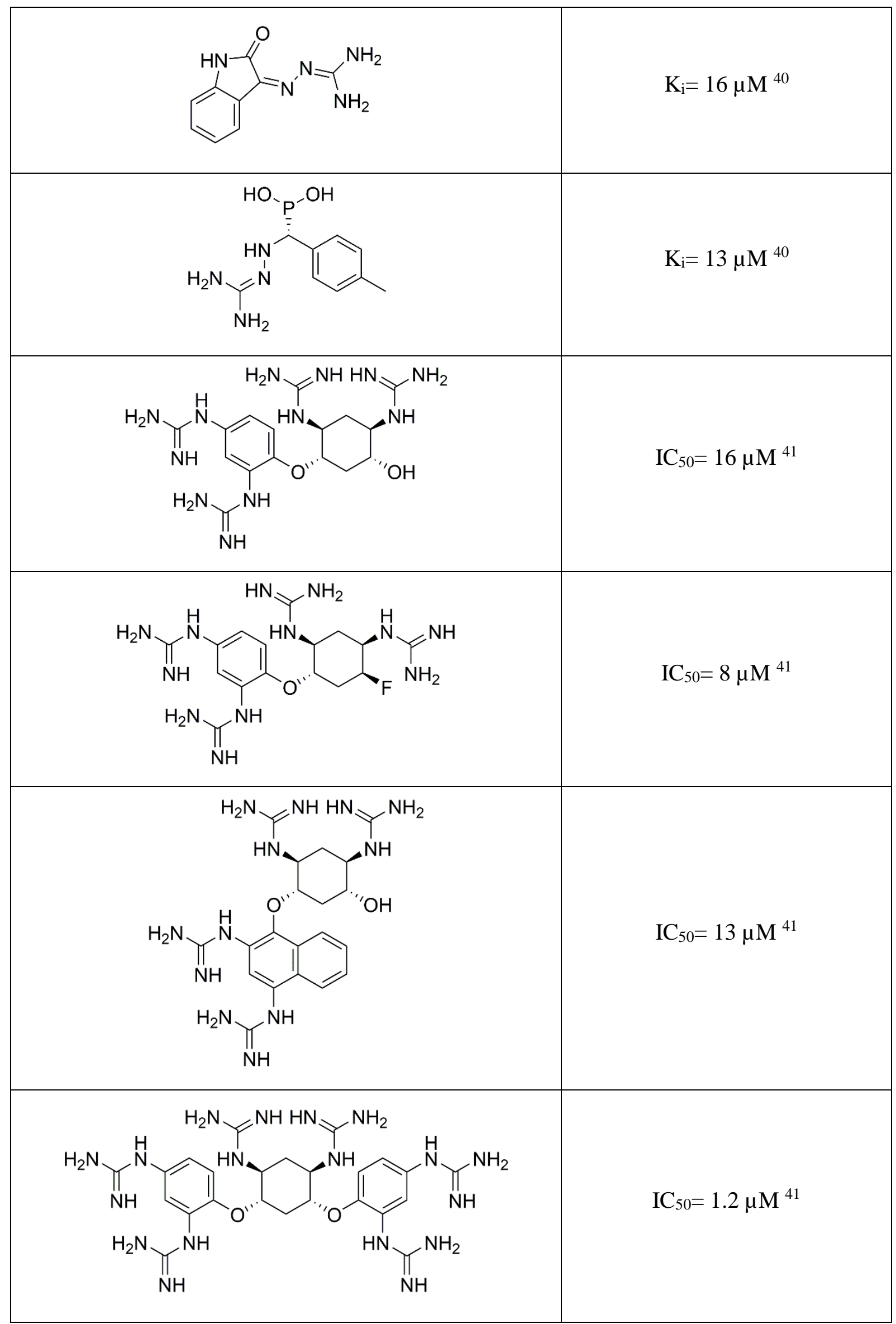




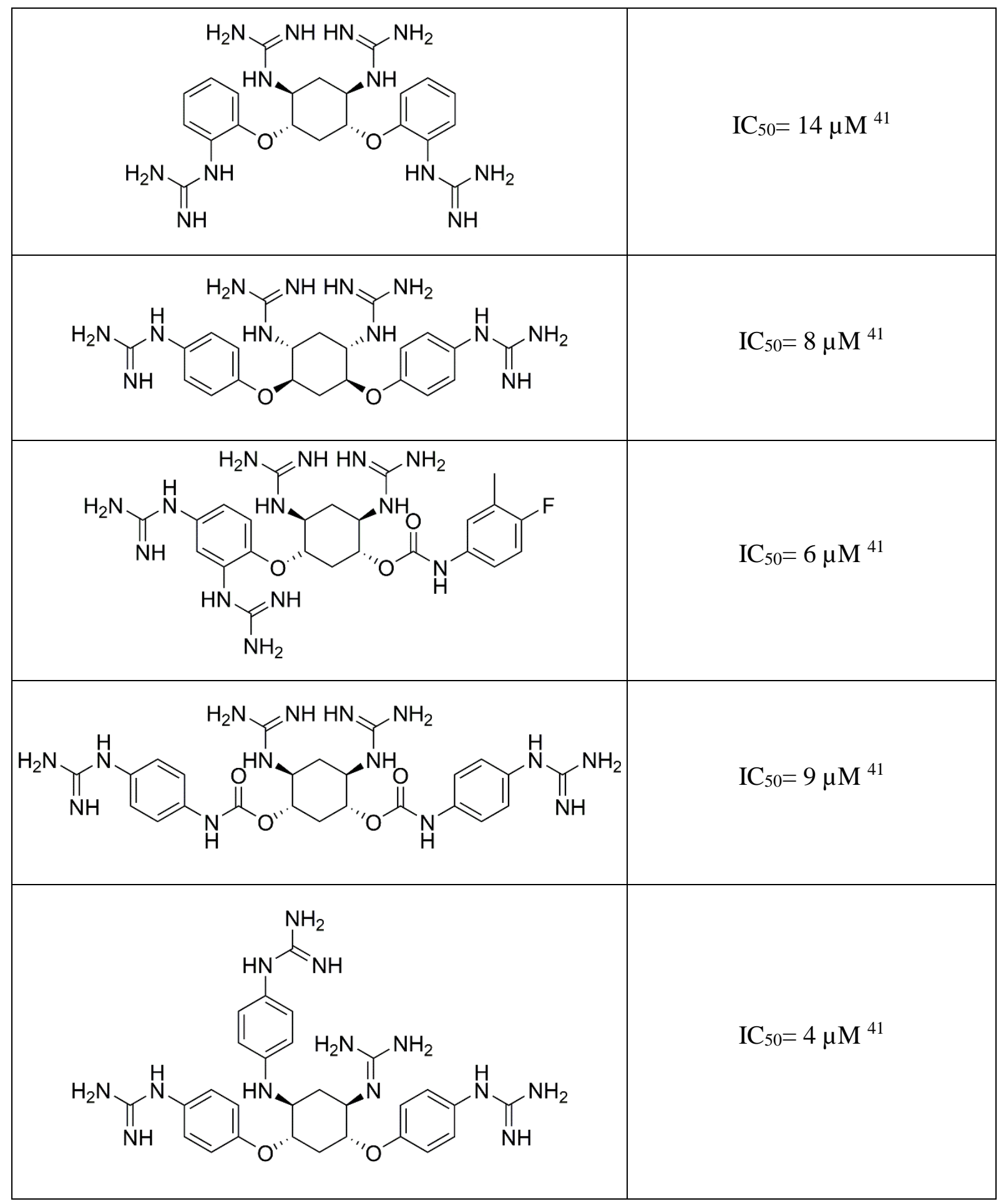




\section{Supplemental Figures}

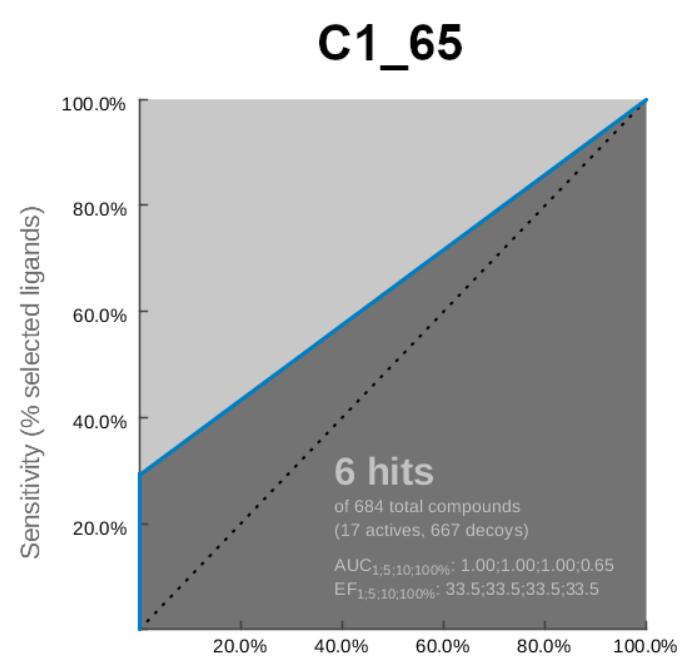

1 - Specificity (\% selected decoys)

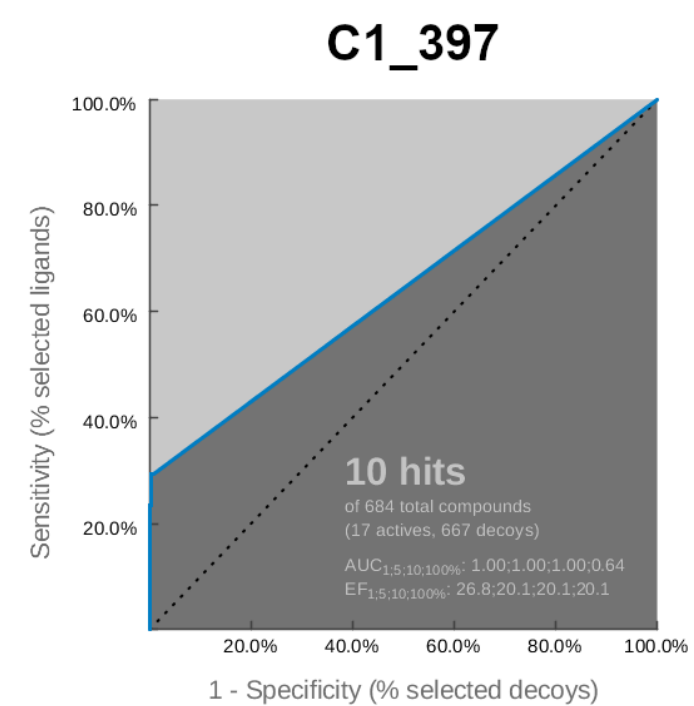

1 - Specificity (\% selected decoys)

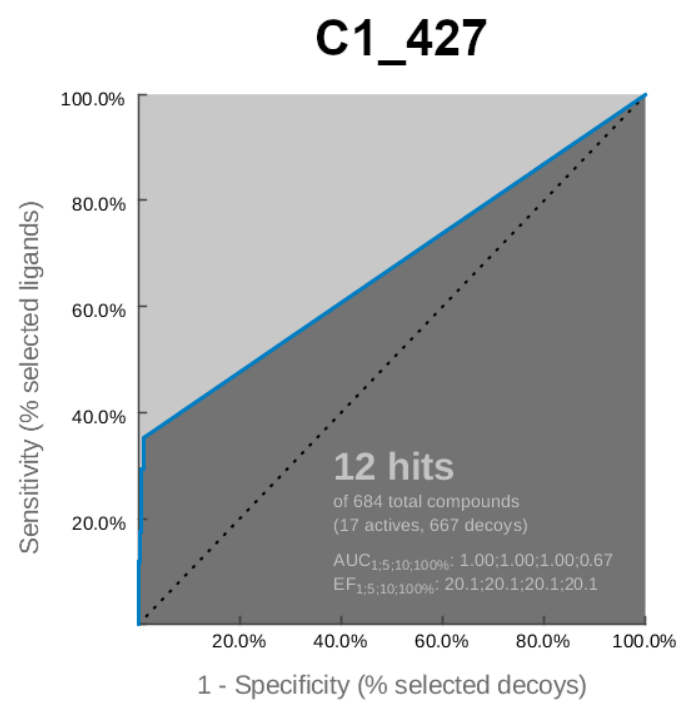

Figure S1. Receiver Operating Characteristic (ROC) Curves for West-Nile virus protease pharmacophores C1_65, C1_397 and C1_427. 


\section{A: ZIKV}
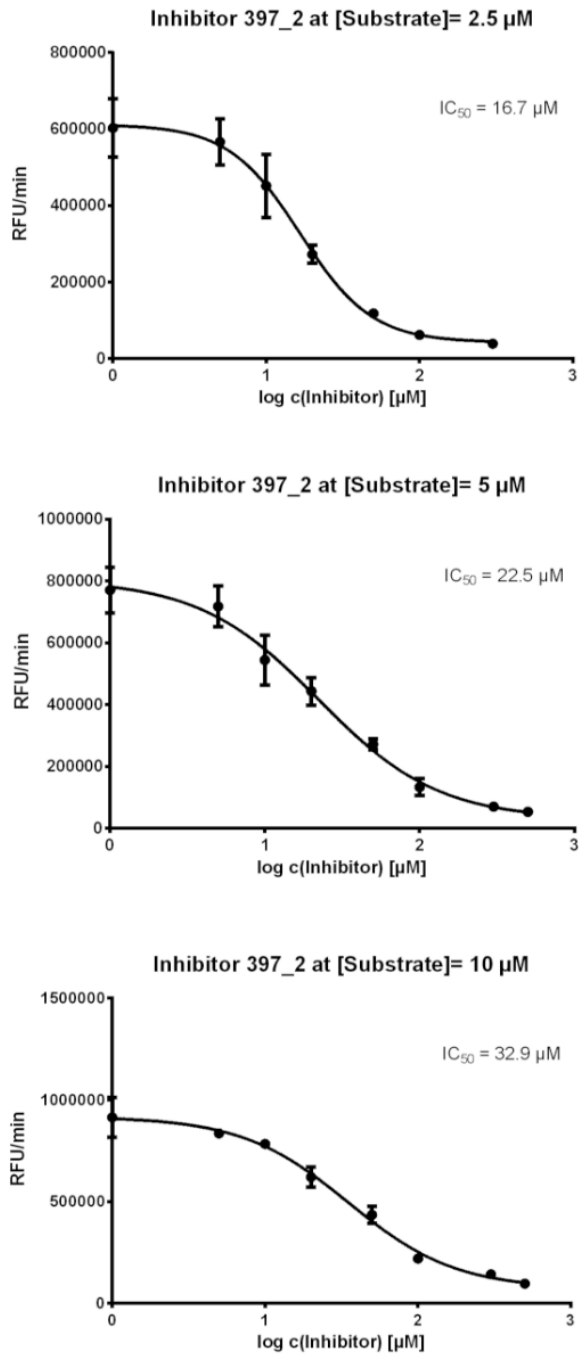

Inhibitor 4271 at [Substrate] $=5 \mu \mathrm{M}$

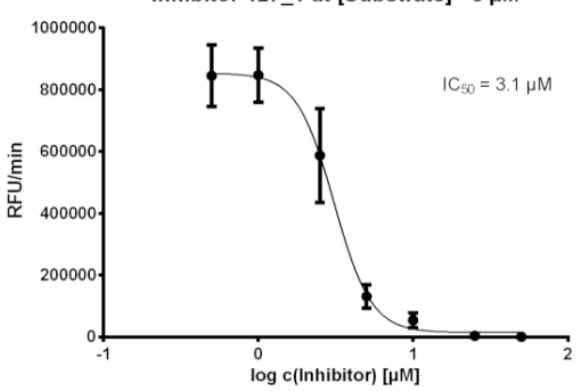

Inhibitor 4271 at [Substrate] $=10 \mu \mathrm{M}$

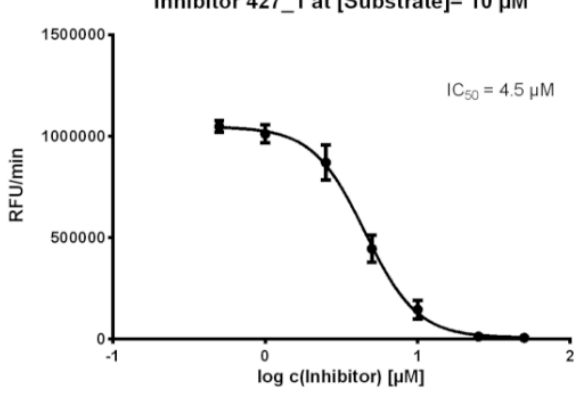

Inhibitor 427 at [Substrate] $=20 \mu \mathrm{M}$

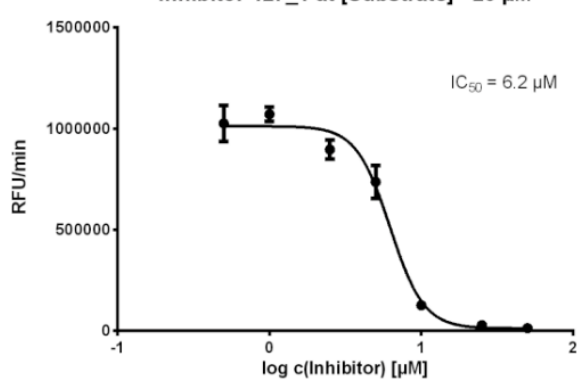




\section{B: WNV}

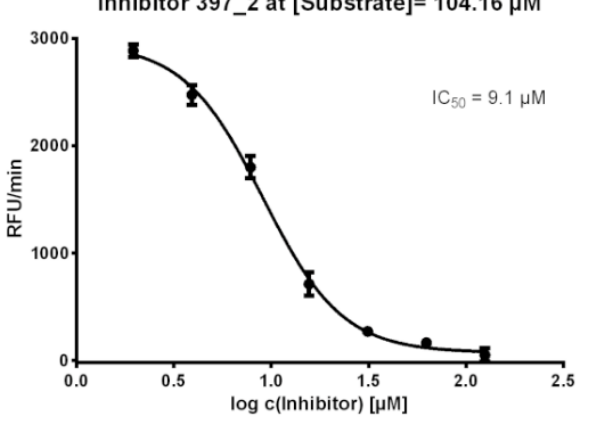

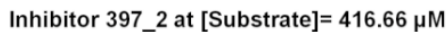

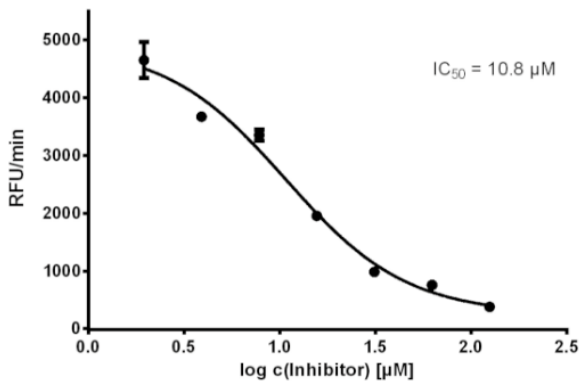

Inhibitor $397 \_$at [Substrate] $=833.33 \mu \mathrm{M}$

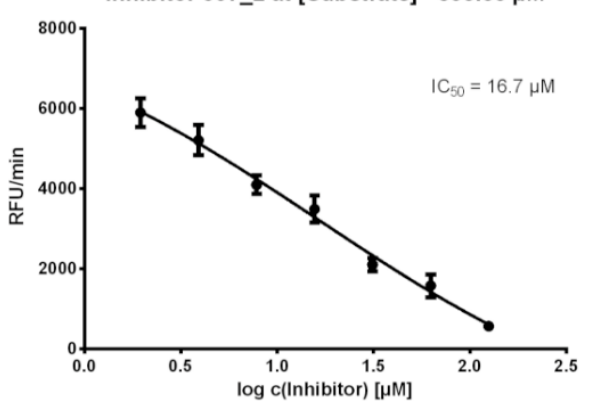

Inhibitor 427_1 at [Substrate] $=52.08 \mu \mathrm{M}$

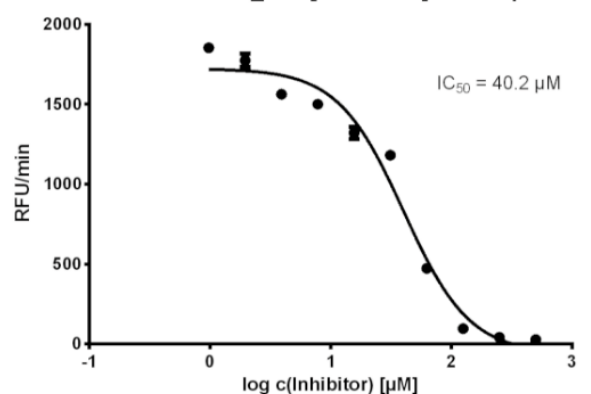

Inhibitor $427 \_1$ at [Substrate] $=208.33 \mu \mathrm{M}$

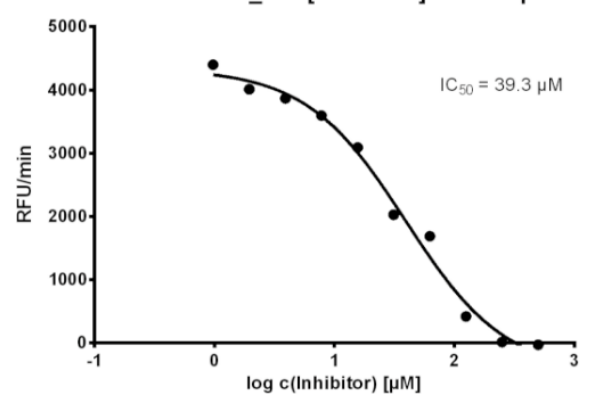

Inhibitor 427_1 at [Substrate] $=833.33 \mu \mathrm{M}$

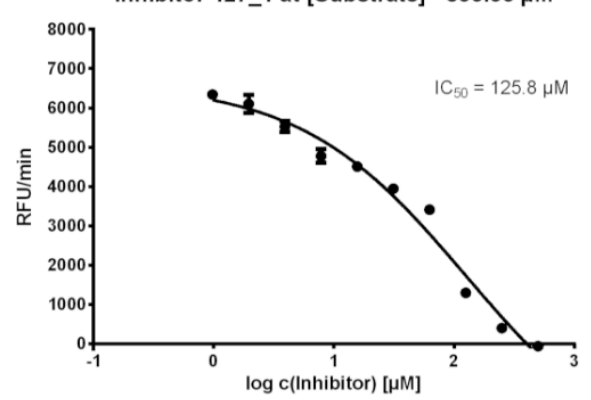




\section{C: DENV2}

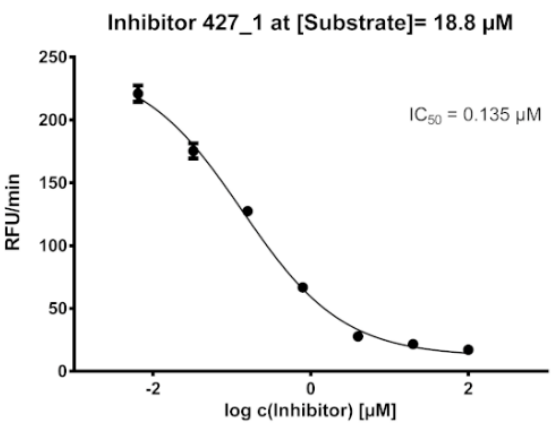

Inhibitor $427 \_1$ at [Substrate] $=37.5 \mu \mathrm{M}$

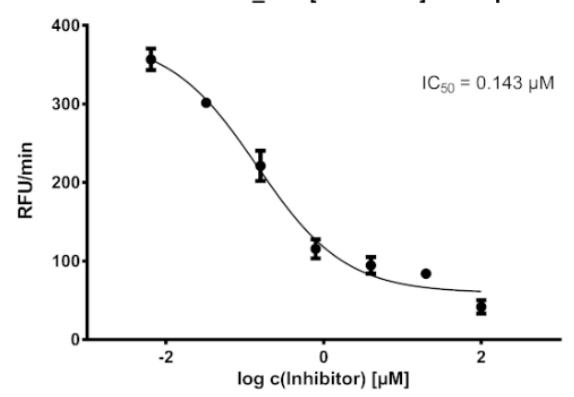

Inhibitor $427 \_1$ at [Substrate] $=75 \mu \mathrm{M}$

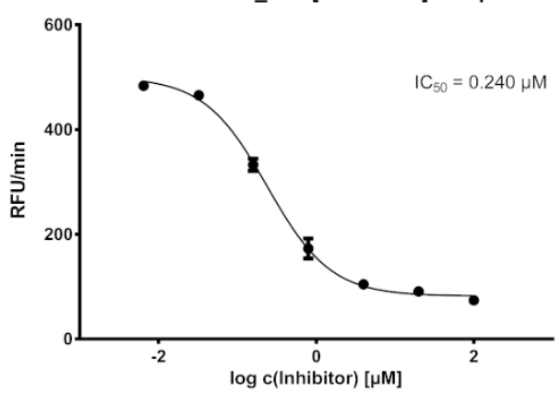

Figure S2. Concentration-response curves used for $K_{i}$ values calculations. A Zika virus protease assay, B West Nile virus protease assay, C Dengue virus serotype 2 protease assay. RFU: Relative fluorescence units. 


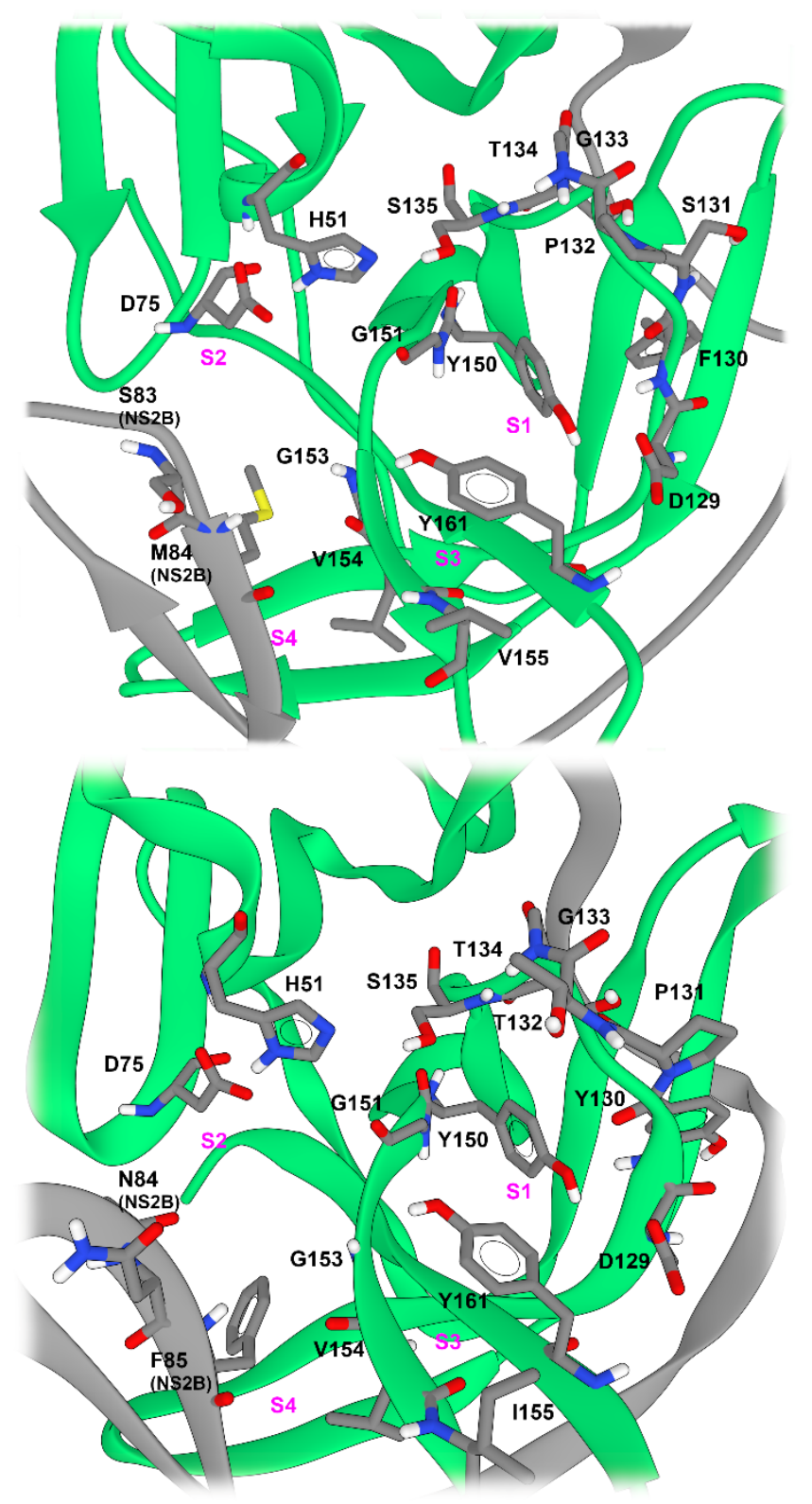

Figure S3. Comparison of DENV2 ${ }^{\text {Pro }}$ (top, homology model) and WNV ${ }^{\text {Pro }}$ (bottom, PDB-ID: 5IDK ${ }^{6}$ ) binding pockets. The key-residues are highlighted with black letters and numbers. Pink letters and numbers indicate protease-sub-pockets. Gray backbone- NS2B, green backbone- NS3. This figure was generated using UCSF Chimera 1.13.1. ${ }^{42}$ 


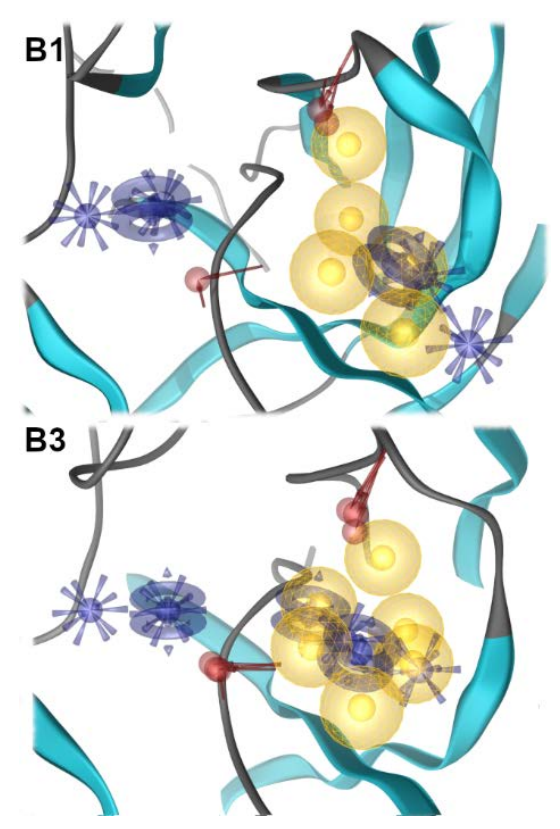

Figure S4. Comparison between focused pharmacophores B1 (WNV ${ }^{\text {Pro }}$ based) and B3 (DENV2 $^{\text {Pro }}$ based) obtained from PyRod analysis of MD simulations (yellow sphereslipophilic contacts, purple rings- aromatic interactions, red arrow- hydrogen bond acceptor, purple star- cationic interaction).
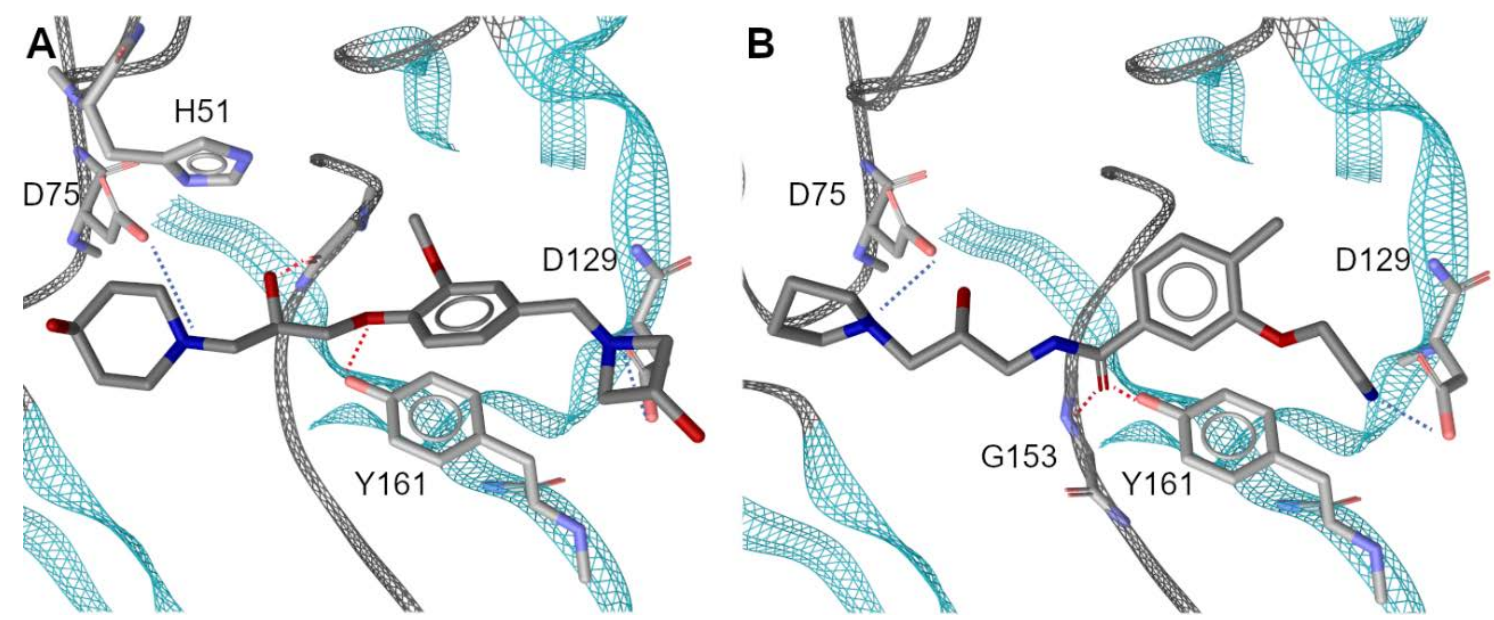

Figure S5. Proposed binding modes for the active inhibitors; compound 397_2 (A) and 427_1 (B) in complex with DENV2 ${ }^{\text {Pro }}$ homology model. Color code: blue lines- ionic contacts, red lines- hydrogen bonds. 

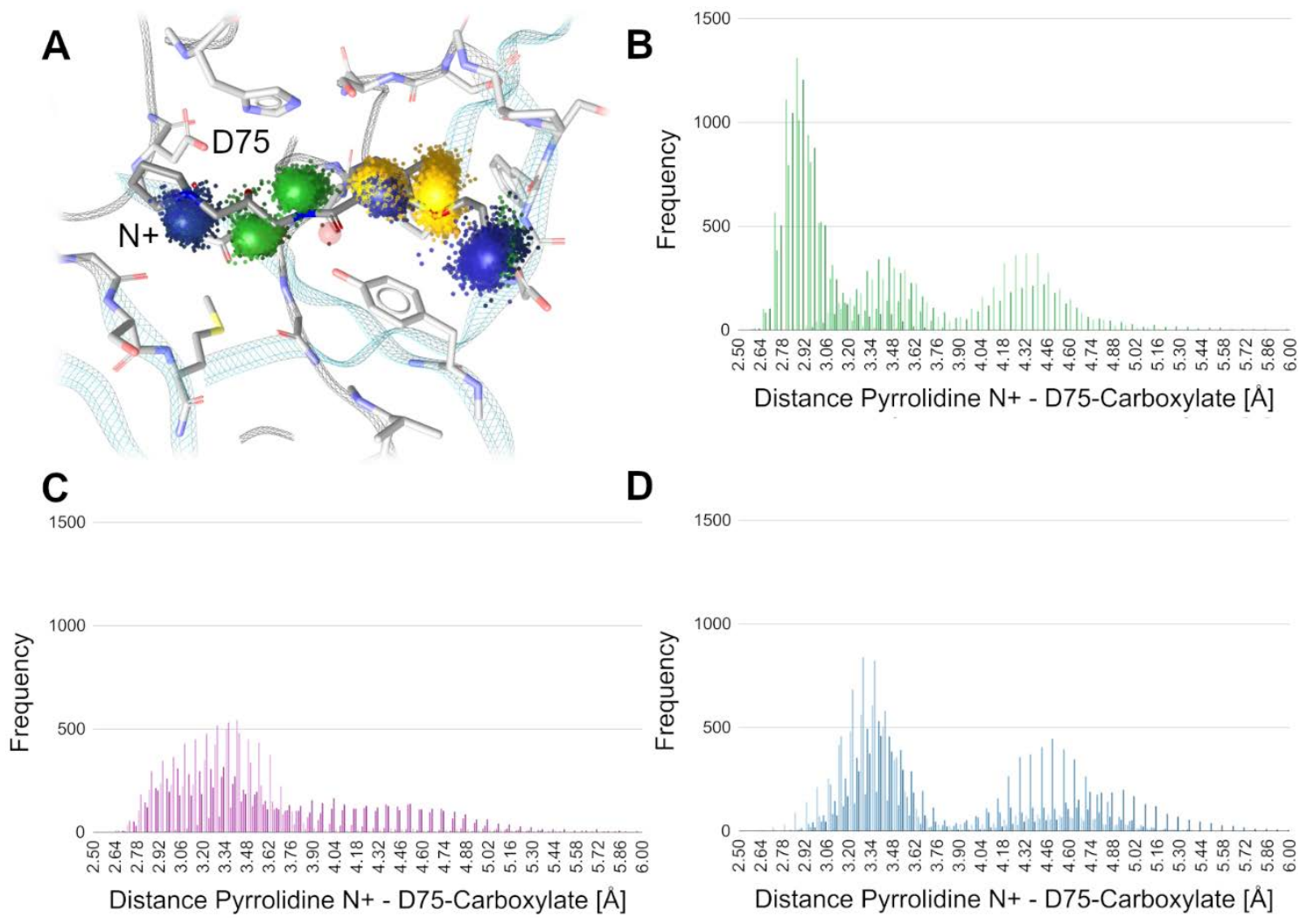

Figure S6. Dynamic pharmacophores generated from MD simulations of compound 427_1 in complex with DENV2 ${ }^{\text {Pro }}$ (A); color code: yellow points- lipophilic contacts, red and green points- hydrogen bond acceptors and donors, respectively, purple points- cationic interactions, blue points- aromatic interactions. Distribution of distances between positively ionizable pyrrolidine moiety in inhibitor 427_1 and carboxylate of D75 in the S2 binding pocket for DENV2 (B, green bars), WNV (C, pink bars) and ZIKV (D, blue bars); different color-shades indicate replicates of MD simulations. 


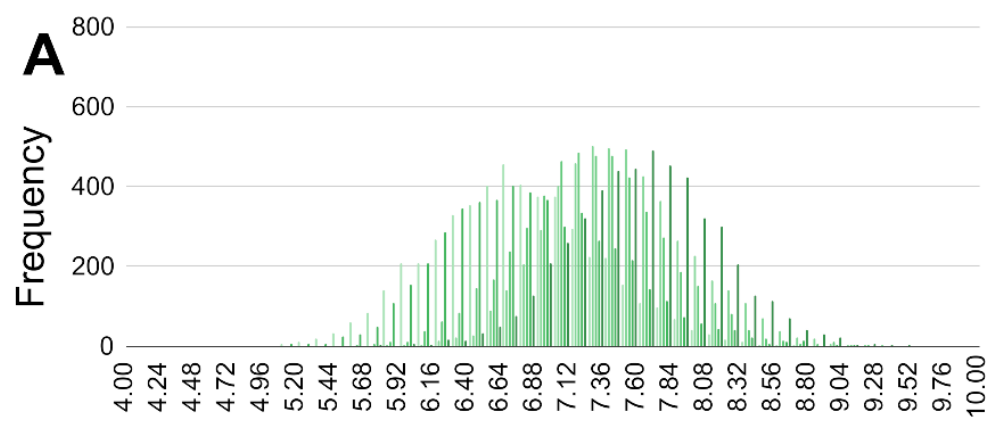

Ca Distance M84 - G153 in DENV2 [Å]

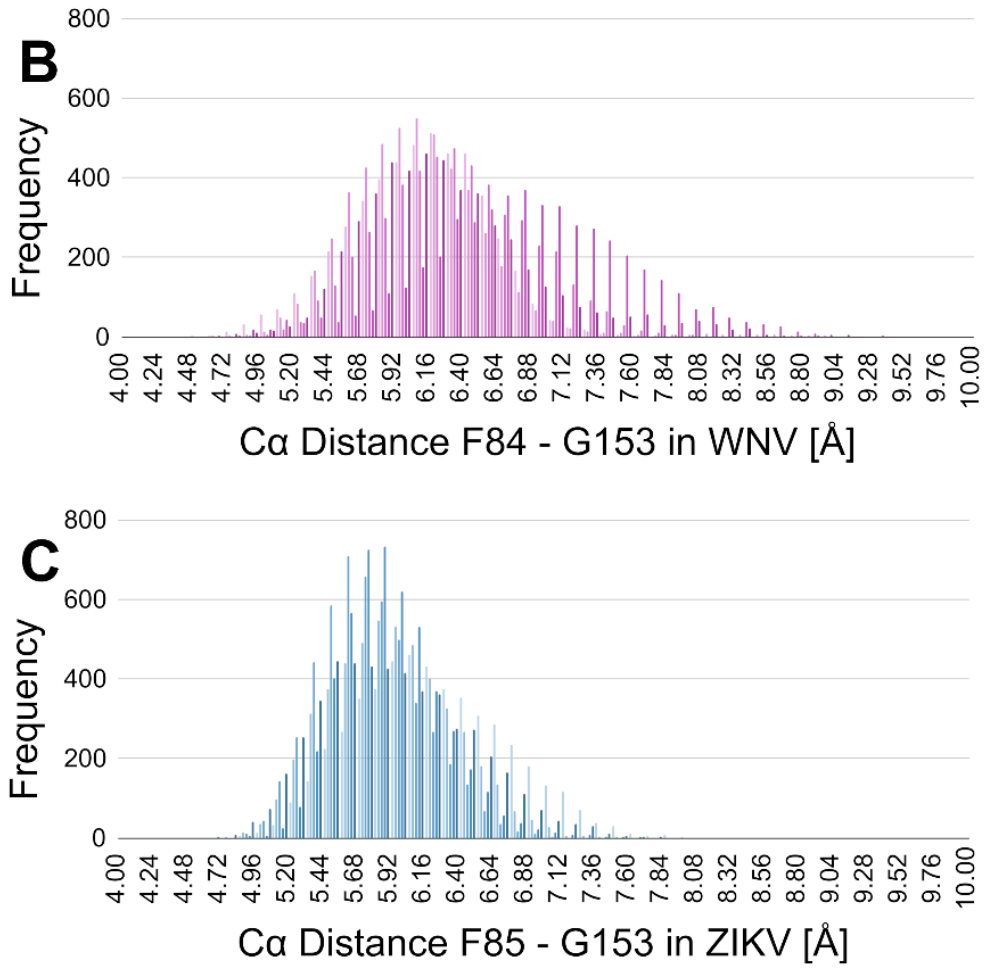

Figure S7. Distribution of distances between C $\alpha$ atoms of residues M84 of NS2B and G153 of NS3 (A, DENV2, green bars), F85 and G153 (B, WNV, pink bars), F84 and G153 (C, ZIKV, blue bars) indicating fitting of protease-conformation to 427_1 in case of DENV2 ${ }^{\text {Pro }}$; different color-shades indicate replicates of MD simulations. 


\section{References}

1. Molecular Operating Environment (MOE), Chemical Computing Group ULC: 1010 Sherbooke St. West, Suite \#910, Montreal, QC, Canada, 2018.

2. Labute, P., The generalized Born/volume integral implicit solvent model: estimation of the free energy of hydration using London dispersion instead of atomic surface area. $J$. Comput. Chem. 2008, 29 (10), 1693-8.

3. Noble, C. G.; Seh, C. C.; Chao, A. T.; Shi, P. Y., Ligand-bound structures of the dengue virus protease reveal the active conformation. J. Virol. 2012, 86 (1), 438-46.

4. Berman, H. M.; Westbrook, J.; Feng, Z.; Gilliland, G.; Bhat, T. N.; Weissig, H.; Shindyalov, I. N.; Bourne, P. E., The Protein Data Bank. Nucleic Acids Res. 2000, 28 (1), 235-42.

5. Li, Y.; Zhang, Z.; Phoo, W. W.; Loh, Y. R.; Li, R.; Yang, H. Y.; Jansson, A. E.; Hill, J.; Keller, T. H.; Nacro, K.; Luo, D.; Kang, C., Structural Insights into the Inhibition of Zika Virus NS2B-NS3 Protease by a Small-Molecule Inhibitor. Structure 2018, 26 (4), 555-564 e3.

6. Nitsche, C.; Zhang, L.; Weigel, L. F.; Schilz, J.; Graf, D.; Bartenschlager, R.; Hilgenfeld, R.; Klein, C. D., Peptide-Boronic Acid Inhibitors of Flaviviral Proteases: Medicinal Chemistry and Structural Biology. J. Med. Chem. 2017, 60 (1), 511-516.

7. Boldescu, V.; Behnam, M. A. M.; Vasilakis, N.; Klein, C. D., Broad-spectrum agents for flaviviral infections: dengue, Zika and beyond. Nat. Rev. Drug. Discov. 2017, 16 (8), 565586.

8. Mysinger, M. M.; Carchia, M.; Irwin, J. J.; Shoichet, B. K., Directory of useful decoys, enhanced (DUD-E): better ligands and decoys for better benchmarking. J. Med. Chem. 2012, 55 (14), 6582-94.

9. Molnet Corina, 3; Molecular Networks GmbH Computerchemie: Nägelsbachstr. 25, 91052 Erlangen, Germany, 2019.

10. Wolber, G.; Langer, T., LigandScout: 3-D pharmacophores derived from proteinbound ligands and their use as virtual screening filters. J. Chem. Inf. Model. 2005, 45 (1), 1609.

11. Wolber, G.; Dornhofer, A. A.; Langer, T., Efficient overlay of small organic molecules using 3D pharmacophores. J. Comput. Aided Mol. Des. 2006, 20 (12), 773-88.

12. Schrödinger Release 2019-2: Maestro, Schrödinger, LLC: New York, NY, 2019.

13. Bowers, K. J. C., E.; Xu, H.; Dror, R. O.; Eastwood, M. P.; Gregersen, B. A.; Klepeis, J. L.; Kolossvary, I.; Moraes, M. A.; Sacerdoti, F. D.; Salmon, J. K.; Shan, Y.; Shaw, D. E. Scalable Algorithms for Molecular Dynamics Simulations on Commodity Clusters Proceedings of the ACM/IEEE Conference on Supercomputing (SC06), Tampa, Florida, 2006.

14. Abascal, J. L.; Vega, C., A general purpose model for the condensed phases of water: TIP4P/2005. J. Chem. Phys. 2005, 123 (23), 234505.

15. Humphrey, W.; Dalke, A.; Schulten, K., VMD: visual molecular dynamics. J. Mol. Graph. 1996, 14 (1), 33-8, 27-8.

16. Schaller, D.; Pach, S.; Wolber, G., PyRod: Tracing Water Molecules in Molecular Dynamics Simulations. J. Chem. Inf. Model. 2019, 59 (6), 2818-2829.

17. Jones, G.; Willett, P.; Glen, R. C.; Leach, A. R.; Taylor, R., Development and validation of a genetic algorithm for flexible docking. J. Mol. Biol. 1997, 267 (3), 727-48.

18. Mooij, W. T.; Verdonk, M. L., General and targeted statistical potentials for proteinligand interactions. Proteins 2005, 61 (2), 272-87.

19. Baxter, C. A.; Murray, C. W.; Clark, D. E.; Westhead, D. R.; Eldridge, M. D., Flexible docking using Tabu search and an empirical estimate of binding affinity. Proteins 1998, 33 (3), 367-82. 
20. Halgren, T. A., Merck molecular force field. I. Basis, form, scope, parameterization, and performance of MMFF94. J. Comput. Chem. 1996, 17 (5-6), 490-519.

21. Halgren, T. A., Merck molecular force field. II. MMFF94 van der Waals and electrostatic parameters for intermolecular interactions. J. Comput. Chem. 1996, 17 (5-6), 520-552.

22. Halgren, T. A., Merck molecular force field. III. Molecular geometries and vibrational frequencies for MMFF94. J. Comput. Chem. 1996, 17 (5-6), 553-586.

23. Halgren, T. A.; Nachbar, R. B., Merck molecular force field. IV. conformational energies and geometries for MMFF94. J. Comput. Chem. 1996, 17 (5-6), 587-615.

24. Halgren, T. A., Merck molecular force field. V. Extension of MMFF94 using experimental data, additional computational data, and empirical rules. J. Comput. Chem. 1996, 17 (5-6), 616-641.

25. Bock, A.; Bermudez, M.; Krebs, F.; Matera, C.; Chirinda, B.; Sydow, D.; Dallanoce, C.; Holzgrabe, U.; De Amici, M.; Lohse, M. J.; Wolber, G.; Mohr, K., Ligand Binding Ensembles Determine Graded Agonist Efficacies at a G Protein-coupled Receptor. J. Biol. Chem. 2016, 291 (31), 16375-89.

26. Sydow, D. Dynophores: Novel Dynamic Pharmacophores. Implementation of Pharmacophore Generation Based on Molecular Dynamics Trajectories and Their Graphical Representation. Master Thesis, Humboldt University, Berlin, 2015.

27. Zhang, Z.; Li, Y.; Loh, Y. R.; Phoo, W. W.; Hung, A. W.; Kang, C.; Luo, D., Crystal structure of unlinked NS2B-NS3 protease from Zika virus. Science 2016, 354 (6319), 15971600 .

28. Lei, J.; Hansen, G.; Nitsche, C.; Klein, C. D.; Zhang, L.; Hilgenfeld, R., Crystal structure of Zika virus NS2B-NS3 protease in complex with a boronate inhibitor. Science 2016, 353 (6298), 503-5.

29. GraphPad Prism, 6.01 for Windows; La Jolla, California USA, 2018.

30. Yung-Chi, C.; Prusoff, W. H., Relationship between the inhibition constant (KI) and the concentration of inhibitor which causes 50 per cent inhibition (I50) of an enzymatic reaction. Biochemical Pharmacology 1973, 22 (23), 3099-3108.

31. Shiryaev, S. A.; Farhy, C.; Pinto, A.; Huang, C. T.; Simonetti, N.; Elong Ngono, A.; Dewing, A.; Shresta, S.; Pinkerton, A. B.; Cieplak, P.; Strongin, A. Y.; Terskikh, A. V., Characterization of the Zika virus two-component NS2B-NS3 protease and structure-assisted identification of allosteric small-molecule antagonists. Antiviral Res. 2017, 143, 218-229.

32. Li, J.; Lim, S. P.; Beer, D.; Patel, V.; Wen, D.; Tumanut, C.; Tully, D. C.; Williams, J. A.; Jiricek, J.; Priestle, J. P.; Harris, J. L.; Vasudevan, S. G., Functional profiling of recombinant NS3 proteases from all four serotypes of dengue virus using tetrapeptide and octapeptide substrate libraries. J. Biol. Chem. 2005, 280 (31), 28766-74.

33. Mueller, N. H.; Yon, C.; Ganesh, V. K.; Padmanabhan, R., Characterization of the West Nile virus protease substrate specificity and inhibitors. Int. J. Biochem. Cell Biol. 2007, 39 (3), 606-14.

34. Nall, T. A.; Chappell, K. J.; Stoermer, M. J.; Fang, N. X.; Tyndall, J. D.; Young, P. R.; Fairlie, D. P., Enzymatic characterization and homology model of a catalytically active recombinant West Nile virus NS3 protease. J. Biol. Chem. 2004, 279 (47), 48535-42.

35. Nitsche, C., Proteases from dengue, West Nile and Zika viruses as drug targets. Biophys. Rev. 2019, 11 (2), 157-165.

36. Bissantz, C.; Kuhn, B.; Stahl, M., A medicinal chemist's guide to molecular interactions. J. Med. Chem. 2010, 53 (14), 5061-84.

37. Ekonomiuk, D.; Su, X. C.; Ozawa, K.; Bodenreider, C.; Lim, S. P.; Yin, Z.; Keller, T. H.; Beer, D.; Patel, V.; Otting, G.; Caflisch, A.; Huang, D., Discovery of a non-peptidic 
inhibitor of west nile virus NS3 protease by high-throughput docking. PLoS Negl. Trop. Dis. 2009, 3 (1), e356.

38. Ekonomiuk, D.; Su, X. C.; Ozawa, K.; Bodenreider, C.; Lim, S. P.; Otting, G.; Huang, D.; Caflisch, A., Flaviviral protease inhibitors identified by fragment-based library docking into a structure generated by molecular dynamics. J. Med. Chem. 2009, 52 (15), 4860-8.

39. Bodenreider, C.; Beer, D.; Keller, T. H.; Sonntag, S.; Wen, D.; Yap, L.; Yau, Y. H.; Shochat, S. G.; Huang, D.; Zhou, T.; Caflisch, A.; Su, X. C.; Ozawa, K.; Otting, G.; Vasudevan, S. G.; Lescar, J.; Lim, S. P., A fluorescence quenching assay to discriminate between specific and nonspecific inhibitors of dengue virus protease. Anal. Biochem. 2009, 395 (2), 195-204.

40. Ganesh, V. K.; Muller, N.; Judge, K.; Luan, C. H.; Padmanabhan, R.; Murthy, K. H., Identification and characterization of nonsubstrate based inhibitors of the essential dengue and West Nile virus proteases. Bioorg. Med. Chem. 2005, 13 (1), 257-64.

41. Cregar-Hernandez, L.; Jiao, G. S.; Johnson, A. T.; Lehrer, A. T.; Wong, T. A.; Margosiak, S. A., Small molecule pan-dengue and West Nile virus NS3 protease inhibitors. Antivir. Chem. Chemother. 2011, 21 (5), 209-17.

42. Pettersen, E. F.; Goddard, T. D.; Huang, C. C.; Couch, G. S.; Greenblatt, D. M.; Meng, E. C.; Ferrin, T. E., UCSF Chimera--a visualization system for exploratory research and analysis. J. Comput. Chem. 2004, 25 (13), 1605-12. 